\title{
\#meetTheMAGICians: Science Communication and visibility of young researchers
}

\section{J. van Scherpenberg, ${ }^{a, *}$ A. Lopez-Oramas, ${ }^{b}$ M. Manganaro ${ }^{c}$ and J. Strišcović ${ }^{d}$ on behalf of the MAGIC Collaboration}

${ }^{a}$ Max-Planck-Institut für Physik

D-80805 München, Germany

${ }^{b}$ Instituto de Astrofísica de Canarias and Dpto. de Astrofísica, Universidad de La Laguna

E-38200, La Laguna, Tenerife, Spain

${ }^{c}$ Croatian MAGIC Group: University of Rijeka, Department of Physics

51000 Rijeka, Croatia

${ }^{d}$ Croatian MAGIC Group: Josip Juraj Strossmayer University of Osijek, Department of Physics 31000 Osijek, Croatia

E-mail: jvsch@mpp.mpg.de

Among the many activities organized by the Outreach working group of the MAGIC (Major Atmospheric Gamma-ray Imaging Cherenkov) Collaboration, we would like to present the ongoing project \#meetTheMAGICians. Under this hashtag, used on our social media pages (@MAGICtelescopes), we collect live streaming events on astroparticle physics topics, series of social media posts, videos and other contents. In addition to increasing the visibility of the MAGIC collaboration, a central goal of \#meetTheMAGICians is to strongly connect the communication of our science to the individual achievements of our researchers. It is a community-wide challenge to increase the individual recognition of early career scientists in large international science collaborations. In this project, we give young members of the MAGIC collaboration the chance to increase their visibility in the astroparticle community by highlighting their individual contributions to our research. At the same time, we aim to communicate to the general public how exciting and diverse astroparticle physics can be, and to stimulate in young students the curiosity towards the extreme Universe. We will present an overview on the present status of the project, and an analysis of both successes and remaining challenges.

\footnotetext{
*** 37th International Cosmic Ray Conference (ICRC2021), ***

*** 12-23 July 2021 ***

*** Berlin, Germany - Online ***
}

\footnotetext{
${ }^{*}$ Presenter
} 


\section{Introduction}

The MAGIC collaboration has a well organized outreach group with around 15 active members. The outreach group takes care of various activities, such as press releases of new results but also the organization of lectures, masterclasses or live tours of the MAGIC telescope's site for students. In the last few years, we have extended our presence on social media. We have had profiles on Facebook and Twitter for a number of years, but have been creating more regular content in the last years. In 2020, we additionally created an Instagram account and started to produce videos for our YouTube channel on a more regular basis.

\section{Aims}

With the project \#meetTheMAGICians we aim to combine two important aspects of the current academic culture. On the one hand, communicating science to the general public has been receiving increasing attention over the last years and has become an important part of research. Our goal is to explain our research to the general public by giving them insights into the operations of our instrument and explaining the physics behind our observations and our latest publications. On the other hand, the astroparticle physics community is facing the challenge of giving enough visibility to individual early career researchers (ECRs) that are part of large international science collaborations. We want to create a platform for young members of our collaboration to highlight their personal contributions to our publications and to science in general.

\section{Methods}

We use the hashtag \#meetTheMAGICians on all our social media channels - Twitter, Facebook, Instagram and YouTube. Under this hashtag we collect content that portraits the work of members of the MAGIC collaboration that are in early stages of their academic career. For special occasions, as for exmaple the Dark Matter Day, we collect a large number of tweets, highlighting all the ECRs that work on the particular topic. The biggest portion of this project are live events that we stream on YouTube, Facebook and Twitter. Since July 2020 we have organized six events to which we invited young members of the MAGIC collaboration mostly at the PhD or Postdoc level. The live streams are organized either to announce a new physics results that has recently been published or to celebrate special occasions, for example the anniversary of a major discovery by the MAGIC collaboration. A member of the MAGIC outreach group acts as the host and talks with the invited experts for about one hour. We introduce our telescopes and the general research of our collaboration and then go deeper into detail of the specific result that we want to promote. We take the time to highlight the individual contributions of the ECRs to the specific result and science in general. Additionally, we also discuss different aspects of their daily lives - the daily struggles with analyzing data, going on observing shifts at the telescopes' site in La Palma, Spain, and additional tasks and responsibilities they take on inside the collaboration. In the end we answer questions asked in the chat by the audience. We keep a relaxed, informal atmosphere during the live events. We show that our jobs can be a lot of fun, while at the same time we are not hiding that we also sometimes run into problems or make mistakes. With this we hope to make our science and us 
scientists approachable to the public, to spark excitement about our research, and to motivate young students to start a physics career.

\section{Reception}

Our twitter campaigns reach around 1000-2000 people, with 50-100 interactions and around 5 retweets or likes per tweet. The live events are advertised on all our social media channels. On Instagram we use posts as well as stories to announce the live streams on which we receive around 200 views and 30-40 likes. The live streams attract a live audience of roughly 60 viewers on YouTube and Facebook that ask around 5 questions per event. After the stream, we upload the videos to our YouTube channel, where they get additional views. The videos have around 300 views in total. In general we get a very positive feedback from the live audience.

\section{Challenges}

While we are happy with the reception of our project, given the fact that it only has been running for one year, we would still like to improve our events and attract a larger audience. However, we face the challenge of having to manage the different time zones that our invited experts live in. This makes it difficult to find a perfect time during the day that works for all speakers as well as being an accommodating time for potential viewers. Additionally, the events and social media campaigns are entirely run on a voluntary basis by full time researchers. That leaves only a limited time to be put into the preparation of these events.

\section{Acknowledgments}

We acknowledge the support from the agencies and organizations listed here:

https://magic.mpp.mpg.de/acknowledgments_ICRC2021 